\title{
Soft QCD Results from CMS
}

\section{Yuan CHAO*i}

National Taiwan University

E-mail: yuanchao@cern.ch

Studies of hadron production in proton-proton collisions, including charged particle transverse momentum, pseudo-rapidity and event-by-event multiplicity distributions at $\sqrt{s}=0.9,2.36$ and 7 $\mathrm{TeV}$ are shown. Measured spectra of identified strange hadrons, reconstructed based on their decay topology, are also presented. Comparisons to several QCD Monte Carlo models and tunes are discussed. Results on two-particle angular correlations over a broad range of pseudo-rapidity and azimuthal angle in p-p collisions are presented at both $\sqrt{s}=0.9$ and $7 \mathrm{TeV}$. In high multiplicity events, a pronounced structure emerges in the two-dimensional correlation function for particle pairs with intermediate transverse momentum of 1-3 GeV/c. Furthermore, Bose-Einstein correlations between identical particles are measured in samples of proton-proton collisions at $\sqrt{s}=0.9$ and $7 \mathrm{TeV}$. Finally, a measurement of the underlying activity in scattering processes with a $p_{T}$ scale in the several $\mathrm{GeV}$ region is also presented.

The 2011 Europhysics Conference on High Energy Physics-HEP 2011,

July 21-27, 2011

Grenoble, Rhône-Alpes France

\footnotetext{
*Speaker.

$\dagger$ On behalf of CMS Collaboration.
} 


\section{Introduction}

The frontier energy scale of the Large Hadron Collider (LHC) provides us a new tool to probe the physics at the new energy regime. With the general purpose Compact Muon Solenoid (CMS) detector [1], one can study the underlying physics as well as have better constraint on the phenomenological models with various quantum chromodynamics (QCD) basic properties measurements. These studies are essential to the searches of Higgs, SUSY and new physics as they provide solid foundations to the understanding of the backgrounds.

The properties of proton-proton collisions are typically described either empirically or with phenomenological models. For the Monte Carlo (MC) simulation, event generator models are based on the assumption of parton hadronization produced via the fragmentation of color strings. These models are based on a convolution of parton distribution functions, the hard-scattering cross section from perturbative calculations, and fragmentation functions. PYTHIA [2] and other general MC generators further introduce multiple parton interaction (MPI) to account for these contributions. The parametrization models as well as different showering schemes are the MC "tunings" to be verified with experimental results at the highest energy to date.

\section{Low $p_{T}$ QCD Measurements}

The soft QCD studies at CMS make use of the measurements on charged particles. The full solid state tracking system of CMS gives a great niche on these studies for its precision. The CMS tracker consists of 1440 silicon pixel and 15148 silicon strip detector modules, immersed in the uniform $3.8 \mathrm{~T}$ magnetic field provided by a $6 \mathrm{~m}$ diameter super-conducting solenoid. The $p_{T}$ resolution for $1 \mathrm{GeV} / c$ charged particles is between $0.7 \%$ at $|\eta|=0$ and $2 \%$ at $|\eta|=2.5$.

One can classify the soft collisions into: elastic scattering, inelastic single-diffractive (SD) dissociation, double-diffractive (DD) dissociation, and inelastic non-diffractive (ND) scattering [3] . The interaction events recorded by CMS are highly dominated by non-single-diffractive (NSD) ones for the CMS trigger picks up mainly ND and DD events and disfavors SD events.

Measurements of primary charged hadron transverse momentum $\left(p_{T}\right)$, pseudo-rapidity $(\eta)$, and the multiplicity distributions $\left(N_{\mathrm{ch}}\right)$ are presented for NSD events in proton-proton collisions at centre-of-mass energies $\sqrt{s}=0.9,2.36$, and $7 \mathrm{TeV}[4,5,6]$. The phase-space-invariant differential yield $E d^{3} N_{\mathrm{ch}} / d p^{3}$ for primary charged particles is compared with an empirical parametrization with the variable $x_{T} \equiv 2 p_{T} / \sqrt{s}$. The CMS results are consistent over the accessible range in $x_{T}$. The ProQ20 tune of PYTHIA 6 shows most consistence at $\sqrt{s}=0.9 \mathrm{TeV}$, while the $7 \mathrm{TeV}$ data are most consistent with PYTHIA 8 at the $10 \%$ level of the full $p_{T}$ range. The measurements of $d N_{\mathrm{ch}} / d \eta$ distributions are obtained, as in Ref. [7], with three methods, based on counting the following quantities: (i) reconstructed clusters in the barrel part of the pixel detector; (ii) pixel track-lets composed of pairs of clusters in different pixel barrel layers; and (iii) tracks reconstructed in the full tracker volume. The third method also allows a measurement of the $d N_{\mathrm{ch}} / d p_{T}$ distribution. The results for the three individual layers within the cluster-counting method are found to be consistent within $1.2 \%$ and combined. Also, the three different measurement methods with agreement to the average within $1 \%$ to $4 \%$ depending on $\eta$ are combined and compared to the measurements of other experiments. The combined result for the central pseudo-rapidity density is higher than 
most predictions and provides new information to constrain ongoing improvements of theoretical models and event generators. Independent emission of single particles yields a Poissonian $P_{n}$, whose magnitude increases with $\sqrt{s}$ [8]. The mean of the multiplicity distribution, $\langle n\rangle$, is equal to the integral of the inclusive single-particle density. The averaged transverse momentum of the charged particles, $\left\langle p_{T}\right\rangle$, shows a positive correlation with the event multiplicity. Traditionally, one studies the Koba-Nielsen-Olesen (KNO) scaling [9] by testing the KNO function $\Psi(z)=\langle n\rangle P_{n}$, where $z=n /\langle n\rangle$, on its $s$ dependence and the normalized moments $C_{q}=\left\langle n^{q}\right\rangle /\langle n\rangle^{q}$. From the 7 $\mathrm{TeV}$ result, the change of slope in $P_{n}$ combined with the strong linear increase of $C_{q}$ indicates a clear violation of KNO scaling with respect to lower energies. The productions of $K_{S}^{0}, \Lambda$ and $\Xi^{-}$ particles at $\sqrt{s}=0.9$ and $7 \mathrm{TeV}[10]$ are also studied. The production ratios $N(\Lambda) / N\left(K_{S}^{0}\right)$ and $N\left(\Xi^{-}\right) / N(\Lambda)$ versus rapidity and transverse momentum show no change with the increase of $\sqrt{s}$.

Bose-Einstein correlations have been measured for the first time at the LHC with CMS in p-p collisions at $\sqrt{s}=0.9,2.36$ and $7 \mathrm{TeV}$ [11]. The effective size of the emission region is observed to grow with charged-particle multiplicity and to decrease with $k_{T}$ in events with large multiplicity. On the other hand, long-range azimuthal correlations for $2.0<|\delta \eta|<4.8$ have been studied for 7 $\mathrm{TeV}$ data [12], leading to the first observation of a long-range ridge-like structure at the near-side $(\delta \phi \approx 0)$ in p-p collisions.

\section{Underlying Event Study}

The "Underlying Event" (UE) is everything in a single proton-proton interaction except for the hard scattering component. The UE contributes through MPI as well as beam-beam remnants, concentrated along the beam direction. It is important to have good understanding on the UE properties for either precision measurements of standard model processes or searches for new physics at the frontier energy scale.

The first measurement of UE activity at the LHC has been published with CMS data at $\sqrt{s}=$ $0.9 \mathrm{TeV}$ [13]. MPI activity is expected to increase with the centre-of-mass energy [14, 15]. At a given $\sqrt{s}$, the UE activity is also expected to increase with the interaction hard scale. It will reach a plateau for high scales, which corresponds to an MPI "saturation" effect. The recent published result [16] from CMS measures the UE properties at $\sqrt{s}=7 \mathrm{TeV}$ with the same analysis procedure and updates the $0.9 \mathrm{TeV}$ results with a 30 times larger sample. In this published result, all measurements are fully corrected for detector effects. The UE studies are done with the referencing leading "track-jet", reconstructed with charged particles. The activities in transverse region in azimuthal direction, $60^{\circ}<\delta \phi<120^{\circ}$, are most sensitive to UE. The $p_{T}$ of the leading track-jet is taken as defining the hard scale in the event. The large increase of activity in the transverse region is observed in $N_{\mathrm{ch}}, \Sigma p_{T}$ and the the charged particle $p_{T}$ spectrum up to $N_{\mathrm{ch}}=30, \Sigma p_{T}=35 \mathrm{GeV} / \mathrm{c}$, and $p_{T}=14 \mathrm{GeV} / \mathrm{c}$. The results are compared with various tunes of PYTHIA 6 as well as PYTHIA 8 4C. A good description of distributions at $\sqrt{s}=7 \mathrm{TeV}$ and $\sqrt{s}$ dependence from 0.9 to $7 \mathrm{TeV}$ is from tune $\mathrm{Z} 1$. Tune $\mathrm{Z2}$ and PYTHIA $84 \mathrm{C}$ are also in reasonable agreement with data.

\section{Conclusion}

Comprehensive studies on various soft QCD topics have been done with CMS data collected 
at $\sqrt{s}=0.9,2.36$ and $7 \mathrm{TeV}$. UE analyzed at $\sqrt{s}=0.9$ and $7 \mathrm{TeV}$, unfolded results compared with various MC tunes. These studies can help on the discrimination of different theoretical modeling as well as Monte Carlo tunings at the LHC energy-scale era.

\section{References}

[1] CMS Collaboration, “The CMS experiment at the CERN LHC”, JINST 3 (2008) S08004. doi:10.1088/1748-0221/3/08/S08004.

[2] T. Sjostrand, S. Mrenna and P. Z. Skands, "PYTHIA 6.4 Physics and Manual," JHEP 0605 (2006) 026, arXiv:hep-ph/0603175 [hep-ph].

T. Sjostrand, S. Mrenna and P. Z. Skands, "A Brief Introduction to PYTHIA 8.1," Comput. Phys. Commun. 178, 852 (2008) arXiv:0710.3820 [hep-ph].

[3] W. Kittel and E. A. De Wolf, "Soft Multihadron Dynamics". World Scientific, Singapore, 2005.

[4] CMS Collaboration, "Charged particle transverse momentum spectra in pp collisions at $\sqrt{s}=0.9$, and $7 \mathrm{TeV}$ ”, submitted to JHEP. arXiv:1104.3547v1.

[5] CMS Collaboration, "Transverse-momentum and pseudorapidity distributions of charged hadrons in collisions at $\sqrt{s}=7$ TeV”, Phys. Rev. Lett. 105 (2010). 10.1103/PhysRevLett.105.022002.

[6] CMS Collaboration, "Charged particle multiplicities in pp interactions at $\sqrt{s}=0.9,2.36$, and $7 \mathrm{TeV}$ ", J. High Energy Phys. 01 (2011) 079. doi:10.1103/PhysRevD.18.4120.

[7] CMS Collaboration, "Transverse-momentum and pseudorapidity distributions of charged hadrons in pp collisions at $\sqrt{s}=0.9$ and $2.36 \mathrm{TeV}$ ", JHEP 02 (2010) 041. doi:10.1007/JHEP02(2010)041.

[8] A. Capella and A. Krzywicki, "Unitarity corrections to short range order: long range rapidity correlations”, Phys. Rev. D18 (1978) 4120. doi:10.1103/PhysRevD.18.4120.

[9] Z. Koba, H. B. Nielsen, and P. Olesen, "Scaling of multiplicity distributions in high-energy hadron collisions", Nucl. Phys. B40 (1972) 317. doi:10.1016/0550-3213(72)90551-2.

[10] CMS Collaboration, "Strange Particle Production in pp Collisions at $\sqrt{s}=0.9$ and $7 \mathrm{TeV}$ ", J. High Energy Phys. 05 (2011) 064. doi:10.1007/JHEP05(2011)064

[11] CMS Collaboration, "Measurement of Bose-Einstein Correlations in pp Collisions at $\sqrt{s}=0.9$ and 7 TeV”, J. High Energy Phys. 05 (2011) 029. doi:10.1007/JHEP05(2011)029

[12] CMS Collaboration, "Observation of Long-Range, Near-Side Angular Correlations in Proton-Proton Collisions at the LHC", J. High Energy Phys. (2011) 091. doi:10.1007/JHEP09(2010)091

[13] CMS Collaboration, "First Measurement of the Underlying Event Activity at the LHC with $\sqrt{s}=0.9$ TeV”, Eur. Phys. J. C 70 (2010) 555-572. doi:10.1140/epjc/s10052-010-1453-9

[14] T. Sjostrand and M. van Zijl, "Multiple parton-parton interactions in an impact parameter picture", Phys. Lett. B 188 (1987) 149. doi:10.1016/0370-2693(87)90722-2.

[15] L. Frankfurt, M. Strikman, and C. Weiss, "Transverse nucleon structure and diagnostics of hard parton-parton processes at LHC”, Phys. Rev. D 83 (2011) 054012, arXiv:1009.2559. doi:10.1103/PhysRevD.83.054012.

[16] CMS Collaboration, "Measurement of the Underlying Event Activity at the LHC with $\sqrt{s}=7 \mathrm{TeV}$ and Comparison with $\sqrt{s}=0.9 \mathrm{TeV}$ ", accepted by JHEP. arXiv:1107.0330 